\title{
Predictors of Hepatocellular Carcinoma in Patients with Liver Cirrhosis
}

\author{
Pop Crina*, Kozma B, Drasovean Silvia, Dobru Daniela, Boeriu Alina \\ University of Medicine and Pharmacy Tirgu Mures, Romania, Department of Gastroenterology
}

\begin{abstract}
Background and aim: Hepatocellular carcinoma (HCC) is one of the most important health problem, with an increasing incidence worldwide. The aim of the present study was to determine the factors that predict HCC occurrence in a group of patients with liver cirrhosis developed on various etiologies.

Material and Methods: A total of 282 consecutive patients with liver cirrhosis seen between January 1, 2013 and July 31, 2014 were enrolled in a prospective study. Data from patient's history, physical exams, laboratory tests and abdominal ultrasound were collected.

Results: 282 patients were included in the study with a median age of 59.6 and a male/female ratio of $1.38 / 1 ; 12.41 \%(35)$ were diagnosed with HCC. Alcohol consumption was documented in 19 cases (54.28\%), without statistical association $(p=0.621)$, and $7(20 \%)$ were smokers ( $p=0.403)$. According to etiological factors of liver cirrhosis: 7 (20\%) had background B virus infection, 15 (42.85\%) HCV infection and $11(31.42 \%)$ cases incriminated other causes of cirrhosis (alcohol). Chi2 test identified a statistically significant association between the occurrence of HCC and viral etiology of cirrhosis ( $p=0.001, r=18,830)$. No statistical association was found between Child Pugh scoring of cirrhosis and HCC $(p=0.774)$ and a statistically significant association but inversely proportional was identified between neoplasia and obesity $(p=0.008, r=-0.157)$.

Conlusion: In conclusion the viral etiology of liver cirrhosis is found to be an important risk factor for HCC, and liver cancer was inversely associated with nutritional status.
\end{abstract}

Keywords: hepatocellular carcinoma; cirrhosis; etiology; predictors

Received: 07 December 2014 / Accepted: 14 January 2015

\section{Introduction:}

Hepatocellular carcinoma (HCC) is the sixth most common cancer, the third cause of cancer related death and accounts of $7 \%$ of all cancers. HCC represents more than $90 \%$ of primary liver cancers and is a major global health problem. [1,2,3]

Long-term follow-up studies have demonstrated that liver cirrhosis is the main risk factor for the development of HCC. In fact, HCC arises in a cirrhotic background in up to $90 \%$ of cases [1]; overall, one-third of cirrhotic patients will develop HCC during their lifetime [1,4]. According to these findings, European Association for the Study of the Liver (EASL) has tried to develop an optimal screening program in order to detect early HCC. Currently, EASL guidelines recommend for all patients with liver cirrhosis a screening program for early detection of HCC, a program based on abdominal ultrasound (US) performed twice a year [2]. A recent meta-analysis has showed that US surveillance detected the majority of HCC tumors before the occurrence of obvious clinical symptoms, with a pooled sensitivity of $94 \%$. However, US was less effective for detecting early-stage HCC, with a reported sensitivity of only $63 \%[2,5]$. Because accurate diagnosis of small liver nodules is of paramount importance to reduce mortality and due to the fact that high-risk patients for HCC are cirrhotic patients, differentiated screening programs, based on

Correspondence to: Crina Pop

E-mail: pscrina@yahoo.com etiology, risk factors and laboratory changes are required. Consequently, EASL guidelines recommend further study to assess the risk factors for developing HCC in patients with cirrhosis [2].

The aim of the study was to determine the factors that predict HCC occurrence in a group of patients with liver cirrhosis developed on various etiologies.

Research problem (working hypothesis) was the role of certain factors, such as gender, etiologic factors of cirrhosis, staging by Child-Pugh score, degree of obesity, the presence of diabetes and smoking / non-smoking status, presence /absence of alcohol consumption, as risk factors for hepatocellular carcinoma.

\section{Material and Methods:}

\section{Patients:}

A prospective observational study was conducted over a period of 18 months, from January 1, 2013 until July 31, 2014, and 282 consecutive patients diagnosed with cirrhosis irrespective of etiology were followed, patients hospitalized in Gastroenterology Department, within the Mures County Hospital.

The study protocol included the following information: demographic data (age, gender), the presence / absence of HCC, smoking status, alcohol consumption, etiology of cirrhosis (chronic viral hepatitis B, C, B + D, autoimmune hepatitis, primary biliary cirrhosis, primary sclerosing cholangitis, non-alcoholic steatohepatitis), body mass 
index (BMI), diabetes mellitus (DM). Data from patient's history, physical exams, laboratory tests and abdominal ultrasound were collected and they were divided into two groups: a) cirrhotic patients with HCC and b) cirrhotic patients without HCC.

Liver cirrhosis was assessed based on abdominal ultrasonography examination (nodular liver surface, heterogeneous echotexture, segmental hypertrophy/atrophy-caudate width: right lobe width $>0.65$, portalization of hepatic vein waveform and signs of portal hypertension- enlarged portal vein: $>13 \mathrm{~mm}$, slow portal venous flow $<15 \mathrm{~cm} / \mathrm{sec}$, portal vein thrombosis +/- cavernous transformation, enlarged superior mesenteric vein and splenic vein: $>10 \mathrm{~mm}$, re-canalisation and hepatofugal paraumbilical venous flow, portosystemic collaterals), associated with clinical signs of portal hypertension (ascites, variceal bleeding, thrombocytopenia or hepatic encephalopathy). Diagnosis of HCC was assessed based on imaging techniques (abdominal ultrasound, contrast-enhanced utrasonography, contrast-enhanced MRI or CT scan), using criteria recommended by European Association for the Study of the Liver (EASL). At contrast enhanced ultrasonography, the detection of hypoechoic nodule or heterogeneous nodule, hypervascularity in the arterial phase with washout in the portal venous or delayed phases, are suggestive for HCC.

The ethical approval was granted by the ethics and scientific review committee of the institution according and informed patient consent was taken adequately.

\section{Statistical analysis:}

Statistical analysis was performed using the Statistical Package for Social Sciences (SPSS, version 20, Chicago, IL, USA) and Microsoft Excel. Data were labeled as nominal and were characterized by means of frequencies. The frequencies of nominal variables were compared with a Chisquare test. Differences in the mean or median between groups were analysed using the t test, Mann-Whitney test, when appropriate. The level of statistical significance was set at $\mathrm{p}<0.05$.

\section{Results:}

A total of 282 patients were included in the study with a median age of 59.6 and a male/female ratio of 1.38/1. All these patients were previously diagnosed with liver cirrhosis and 35 of them (12.41\%) were diagnosed with hepatocellular carcinoma (Figure 1, Figure 2). Of all individuals with HCC (35), 25 (71.42\%) were males and 10 (28.57\%) females.

A history of alcohol consumption was documented in 19 cases (54.28\%) of 35 patients with HCC; using univariate analysis, a statistical significant association between de presence of HCC and alcohol intake could not be demonstrated ( $\mathrm{p}=0.621)$. Of 35 patients, $7(20 \%)$ were smokers and to assess whether tobacco smoking is associated with the neoplasia, Chi2 test was performed, without any statistical relevance $(\mathrm{p}=0.403)$.

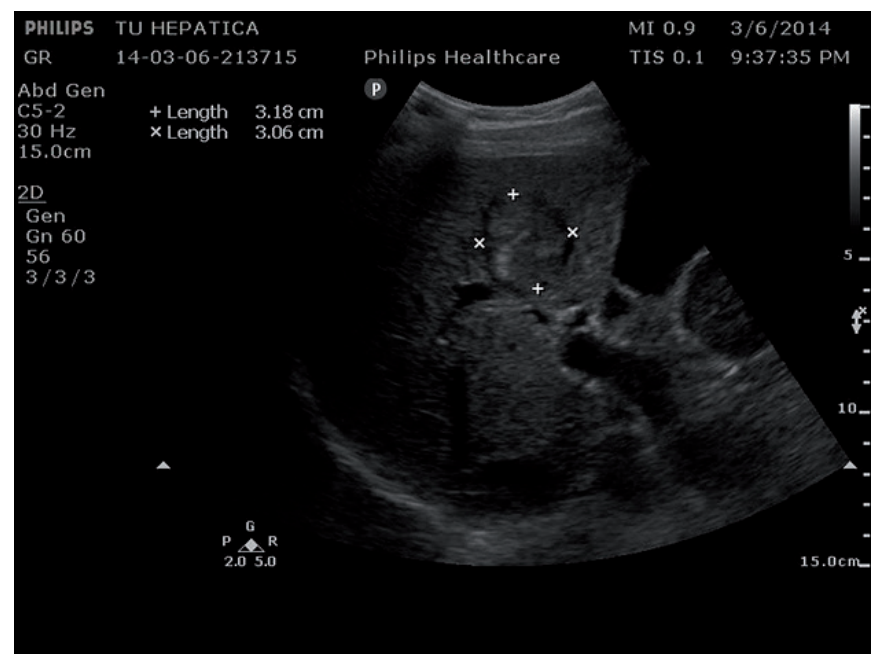

Fig. 1. Abdominal ultrasound examination- Hepatocellular carcinoma

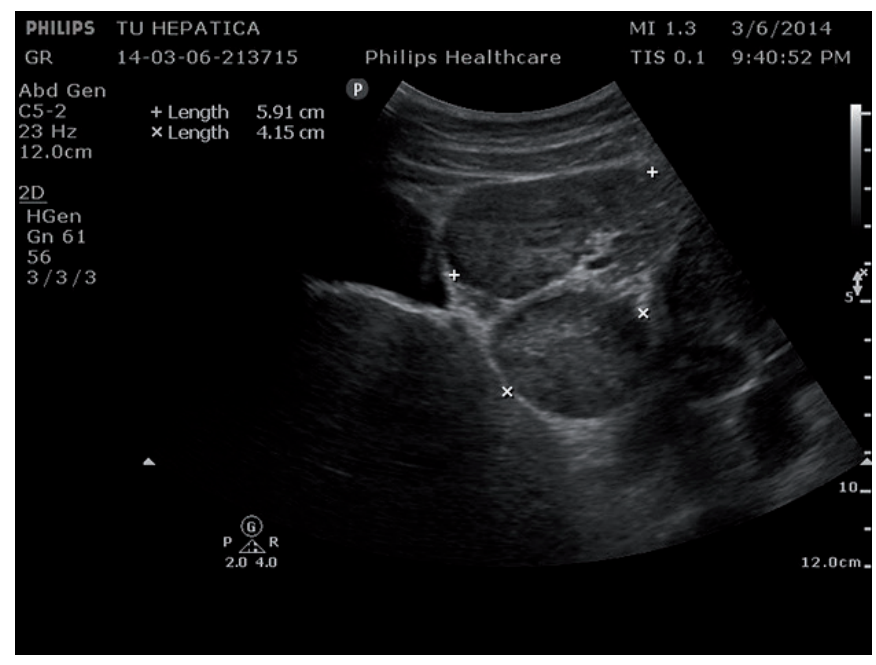

Fig. 2. Abdominal ultrasound examination-multicentric HCC

All 35 patients with HCC were divided according to etiological factors of liver cirrhosis: 7 (20\%) individuals had background B virus infection, 15 (42.85\%) HCV infection and $11(31.42 \%)$ cases incriminated other causes of cirrhosis (alcohol). No cases of HCC have developed on billiary cirrhosis $(\mathrm{PBC})$, primary sclerosing cholangitis (PSC), non-alcoholic steatohepatitis or other autoimmune liver diseases. Chi2 test identified a statistically significant association between the occurrence of HCC and viral etiology of cirrhosis $(\mathrm{p}=0.001, \mathrm{r}=18,830)$. (Table I)

Table I. Etiological factors of liver cirrhosis

\begin{tabular}{ccccc}
\hline & $\begin{array}{c}\text { Group A (HCC+) } \\
\text { N=35 }\end{array}$ & $\begin{array}{c}\text { Group B (HCC-) } \\
\text { N=247 }\end{array}$ & P value \\
\hline VHB & 7 & $(20 \%)$ & $15(6.07 \%)$ & \\
VHB+C & 0 & $(0 \%)$ & $4(1.61 \%)$ & \\
VHB+D & $2(5.71 \%)$ & $5(2.02 \%)$ & 0.01 \\
VHC & $15(42.8 \%)$ & $63(25.5 \%)$ & \\
Non-postviral & $11(31.4 \%)$ & $160(64.7 \%)$ & \\
\hline
\end{tabular}

HCC: Hepatocellular carcinoma, VHB: Viral hepatitis B, VHB+C: Viral hepatitis $B+C, V H B+D$ : Viral hepatitis B+D, VHC: Viral hepatitis C 
Analysis according to the presence or absence of diabetes mellitus (DM) has shown that, of the 35 individuals with HCC 11 (35.42\%) had associated diabetes. A statistically significant association could not be found between these variables $(\mathrm{p}=0.065)$

In the study group, $23.07 \%$ of underweight patients developed HCC, $19.04 \%$ were within the normal range of BMI, $10.46 \%$ were overweight and no obese patient presented HCC. A statistically significant association but inversely proportional was identified between neoplasia and obesity ( $\mathrm{p}=0.008, \mathrm{r}=-0.157$ ).

According to Child Pugh classification of liver cirrhosis, of 35 patients with HCC, 12 (34.28\%) were classified in Child A, 16 (45.71\%) in Child B and 7(20\%) in Child C and no significant association was found between the variables $(\mathrm{p}=0.774)$. (Table II $)$

Table II. Child Pugh classification

\begin{tabular}{cccc}
\hline & $\begin{array}{c}\text { Group A (HCC+) } \\
\text { N=35 }\end{array}$ & $\begin{array}{c}\text { Group B (HCC- } \\
\text { N=247 }\end{array}$ & P value \\
\hline Child A & 12 & 97 & \\
Child B & 16 & 94 & 0.774 NS \\
Child C & 7 & 53 & \\
\hline
\end{tabular}

HCC: hepatocellular carcinoma

\section{Discussion:}

Approximately 90\% of HCCs occurs in patients with chronic liver diseases, most often at the cirrhosis stage. Currently, the major goal to prevent mortality is to detect HCC at an early stage, when curative treatment can be applied. EASL guidelines recommend the surveillance of all patients with liver cirrhosis. Because of the difficulties of early diagnosis, still the surveillance program for cirrhosis remains controversial, as well as the best period of periodicity for surveillance (ranging from every 3 months to every 12 months). The identification of risk factors associated with HCC plays an important key role for any protective strategy against cancer, because there are groups of individuals with increased risk compared to others. Consequently, differentiated screening programs are required based on etiology or risk factors [6,7].

The liver is a sexually dimorphic organ, with differentiation (by gender) of gene expression, mitochondrial function, microsomal enzyme activity, membrane lipid composition and immune response. In males, testosterone release growth hormone from the pituitary gland, which activates tyrosine phosphorylation cascade through JAK2 / STAT5. In women, growth hormone is secreted in smaller quantities. Although Dhir et al. and Waxman have demonstrated this sexual dimorphism, which causes a differentiated liver metabolism of drugs and toxins in women compared to men, and leads to higher incidence of HCC in men, male gender was not found to be associated with HCC in our study $[8,9]$. From a total of 35 patients diagnosed with
HCC, $25(71.42 \%)$ were males. Also, in a report of Bosch et al., men are about three to five times more likely to develop HCC than women [10]. The sexual dimorphism may partially explain the high incidence of HCC among men. Another explanation could be the increased consumption of toxic in male individuals.

Tobacco smoke contains several mutagenic initiating agents, as well as potential promoting agents. Heavy alcohol drinking is likely to have a promoting or growthenhancing effect via the process of cirrhosis. Thus, smoking and alcohol could act together along the same pathway of hepatocarcinogenesis. In a study performed by Kuper et al., a multiplicative, interactive effect of heavy smoking and heavy drinking is demonstrated in the development of HCC [11]. In terms of our study, contrary to the literature, no significant association was found between these two factors and the development of HCC (of the 35 only 19 patients with HCC were alcohol drinkers and 7 smokers).

Although earlier studies denied the association between type II diabetes mellitus (DM) and HCC, recent data from literature emphasize the role of this condition in the development of neoplasia. An association between DM and chronic liver disease has been reported, but the temporal relationship between these conditions remains unknown. Amarapurkar et al. identified in a recent issue of Annals of Hepatology, an association between the presence of diabetes and advanced morphological liver damage [12]. An increased risk of HCC by 2 to 4 -fold in patients with DM, even after adjusting for other predisposing factors, was described by El-Serag and colleagues [13]. When chronic viral hepatitis $\mathrm{C}$ or alcohol intake are associated, the risk of HCC is increased by 10 -fold. It was demonstrated that $\mathrm{DM}$ is a negative prognostic factor for long-term survival in patients with liver cirrhosis and mortality is accelerated by the presence of this condition. Consequently, it can be a decisive work on management strategies in patients with liver disease and diabetes [12-15]. Our study did not demonstrate a significant association between the presence of diabetes in patients with cirrhosis and HCC development. Of 35 patients with HCC, only 11 had diabetes.

The main risk factors in HCC are infections with hepatitis $\mathrm{B}$ and $\mathrm{C}$ viruses. Many studies have shown an association between body mass index (BMI) and liver cancer. Beicheng has reported in a recent number of Journal of Hepatology that BMI represents an independent predictive factor for liver cancer [16]. In our study, an inverse relationship between nutritional status and HCC was found; the explanation may be probably the fact that patients have already suffered significant weight loss, due to the presence of neoplasia and decompensated cirrhosis.

No statistically significant difference was found between the different stages of cirrhosis, but the majority of patients were classified in Child A and B (34.28\% to 45.61\%). Few cases of HCC in individuals classified in Child-Pugh C was revealed in the study, which can probably be explained 
by an increased risk of cirrhosis complications and also the occurrence of death, with disease progression and impaired general condition.

In agreement with data reported in the literature, a significant association between the viral etiology of cirrhosis and HCC occurrence was found in our study. Chronic viral hepatitis $\mathrm{B}$ and $\mathrm{C}$ are major etiological factors of the neoplasia, and the risk is increased by a high level of viral replication [17-19]. Recently, Lok et al. have demonstrated a high incidence of HCC in advanced liver fibrosis caused by chronic hepatitis $\mathrm{C}$, without being influenced by antiviral therapy. Additional studies are required to confirm the occurrence of HCC among patients with bridging fibrosis and to determine whether such patients should undergo HCC surveillance, similar with patients with liver cirrhosis [17]. In the same context, in a population-based study with long term follow-up of inactive HBV carriers, Chen and colleagues showed that inactive carriers still had a significantly increased risk of developing HCC [19].

In this study, no cases of HCC developed on primary biliary cirrhosis or primary sclerosing cholangitis were found, which is explained by the lower incidence of these two pathologies.

The present research has several limitations. The study was primarily limited by its small sample size. The sample size could have been extended by including patients admitted in Internal Medicine Department also. An earlier start in data collection would have increased the time needed to survey more participants.

Conclusions:

In the present study, male gender was not found to be a significant risk factor. A statistically significant difference was observed in the type of cirrhosis: $\mathrm{B}$ viral infection and hepatitis $\mathrm{C}$ are the leading cause of HCC (more than 60\%). Although alcohol and smoking are known risk factors for HCC in this study none significantly increase the risk of HCC. An association between HCC and diabetes was not found. Obesity is a known risk factor for HCC, but our data are inversely correlated with nutritional status, the reason being that at the time of diagnosis, patients have already undergone weight loss.

\section{References:}

1. Brian I. Carr-Tumors of the liver and biliary tree, In: Dan L. Longo MD, Anthony S. Fauci MD, Harrison's Gastroenterology and Hepatology, McGrawHill Education, United States, 2013:535-544.

2. EASL-EORTC- Clinical Practice Guidelines, Journal of Hepatology 2012;56:908-943.

3. National Cancer Institute. PDQ_ levels of evidence for adult and pediatric cancer treatment studies. Bethesda, MD: National Cancer Institute. Date last modified 26/August/2010. <http://cancer.gov/cancertopics/pdq/ levelsevidence-adult-treatment/healthprofessional/>;

4. Grieco A, Pompili, M, Caminiti, G, et al. Prognostic factors for survival in patients with early-intermediate hepatocellular carcinoma undergoing non-surgical therapy: comparison of Okuda, CLIP, and BCLC staging systems in a single Italian centre., Gut, 2005; 54,3:411-418.

5. Bosetti C, Levi F, Boffetta $P$, et al. Trends in mortality from hepatocellular carcinoma in Europe, 1980-2004. Hepatology 2008;48:137-145.

6. Yang HI, Sherman M, Su J, et al. Nomograms for Risk of Hepatocellular Carcinoma in Patients With Chronic Hepatitis B Virus Infection. J Clin Oncol. 2010;28: 2437-2444.

7. Chen JD, Yang HI, lloeje UH, et al. Carriers of Inactive Hepatitis B Virus Are Still at Risk forHepatocellular Carcinoma and Liver-Related Death. Gastroenterology 2010;135:1747-1754.

8. Dhir RN, Dworakowski W, Thangavel C, Shapiro BH. Sexually dimorphic regulation of hepatic isoforms of human cytochrome p450 by growth hormone. J Pharmacol Exp Ther 2006;316:87-94.

9. Waxman DJ, Holloway MG. Centennial perspective: sex differences in the expression of hepatic drug metabolizing enzymes. Mol Pharmacol 2009;76: 215-228.

10. Bosch FX, Ribes J, Diaz M, Cleries R. Primary liver cancer: worldwide incidence and trends. Gastroenterology 2004;127:5(1):S5-S16.

11. Kuper $\mathrm{H}$, Tzonou A, Kaklamani E, et al. Tobacco smoking, alcohol consumption and their interaction in the causation of hepatocellular carcinoma. Int. J. Cancer 2000;85: 498-502.

12. Amarapurkar D, Das HS. Chronic liver disease in diabetes mellitus. Tropical Gastroenterol 2002;23:3-5.

13. El-Serag HB, Tran T, Everhart JE. Diabetes increases the risk of chronic liver disease and hepatocellular carcinoma. Gastroenterology 2004;126:460468.

14. Ruhl CE, Everhart JE. Determinants of the association of overweight with elevated serum alanine aminotransferase activity in the United States. Gastroenterology 2003; 124:71-79.

15. Zen Y, Katayanagi K, Tsuneyama K, et al. Hepatocellular carcinoma arising in non-alcoholic steatohepatitis. Pathol Int 2001;51:127-131.

16. Sun B, Karin M. Obesity, inflammation, and liver cancer. J Hepatol 2012;56:704-713

17. Lok AS, Seeff LB, Morgan TR, et al. Incidence of hepatocellular carcinoma and associated risk factors in hepatitis C-related advanced liver disease. Gastroenterology 2009;136:138-148.

18. Yang HI, Sherman M, Su J, et al. Nomograms for Risk of Hepatocellular Carcinoma in Patients With Chronic Hepatitis B Virus Infection. J Clin Oncol 2010;28: 2437-2444.

19. Chen JD, Yang HI, lloeje UH, et al. Carriers of Inactive Hepatitis B Virus Are Still at Risk forHepatocellular Carcinoma and Liver-Related Death. Gastroenterology 2010;135:1747-1754. 